\title{
TIC en la enseñanza del inglés en 10 institutos educativos bilingües de Tegucigalpa, Honduras en el 2016
}

Geral Ivar Matute ${ }^{1}$

Rosa Palacios Gamez ${ }^{2}$

\section{RESUMEN}

Las Tecnologías de Información y Comunicación tienen una gran influencia en la educación como también en el proceso de enseñanza-aprendizaje de una lengua extranjera. Debido a la importancia de las TIC, fue oportuno analizar el uso de estas herramientas tecnológicas en 10 instituciones bilingües de Tegucigalpa. Tomando en cuenta el conocimiento de los docentes sobre estas tecnologías, las capacidades instaladas en las instituciones en lo que a equipo tecnológico se refiere y el impacto que provocan las TIC en el proceso de enseñanza-aprendizaje del inglés. Alcanzados estos objetivos, se obtuvo que el $74.3 \%$ de los docentes tienen un nivel entre excelente y bueno en el dominio de herramientas tecnológicas. Asimismo, se mostró que el $94.3 \%$ de los centros de educación bilingüe de Tegucigalpa cuentan con los dispositivos tecnológicos necesarios para ensenar la lengua inglesa. De acuerdo a la percepción de docentes y estudiantes, el uso de las TIC juega un papel fundamental en la enseñanza del inglés ya que la realización de actividades lúdicas y la variedad de información que las herramientas tecnológicas ofrecen captan la atención y despierta el interés de los estudiantes. Este diagnóstico permitió proponer algunos lineamientos básicos que se deberían aplicar en la Carrera de Lenguas Extranjeras, UNAH con el fin de preparar a los futuros egresados tomando en cuenta las demandas de las instituciones de educación bilingüe.

Palabras clave: TIC,educación bilingüe, enseñanza, inglés.

\footnotetext{
${ }^{1}$ Estudiante de la Carrera de Lenguas Extranjeras, , Facultad de Humanidades y Artes-UNAH, mgeralivar@yahoo.com

${ }^{2}$ Profesora de la Carrera de Lenguas Extranjeras, Facultad de Humanidades y Artes-UNAH
} 


\section{ABSTRACT}

Information and Communication Technologies have a great influence on education as well as on the teaching process learning a foreign language. Due to the importance of the ICT, it was opportune to analyze the use of these technological tools in the bilingual institutions of Tegucigalpa taking into account the knowledge of the teachers about these technologies, the installed capacities in the institutions as far as technological equipment is concerned and The impact of ICT on the process of Teaching Learning English. Achieving these objectives, it was obtained that $74.3 \%$ of teachers have a level between excellent and good in the domain of technological tools. Likewise, it was shown that $94.3 \%$ of the bilingual education centers in Tegucigalpa have the necessary technological devices to teach the English language. According to the perception of teachers and students, the use of ICT plays a fundamental role in the teaching of English since the realization of play activities and the variety of information that the technological tools offer capture the attention and awakens the interest of the students. This diagnosis allowed to propose some basic guidelines that should be applied in the Race of Foreign Languages, UNAH in order to prepare the future graduates taking into account the demands of the institutions of bilingual education.

Keywords: ICT, bilingual education, teaching, English. 


\section{INTRODUCCIÓN}

El inglés es una de las lenguas más habladas a nivel mundial debido al auge de la globalización en las últimas décadas, de igual manera en Honduras el número de personas que hablan el inglés como segunda lengua o como lengua extranjera continua creciendo. Se puede apreciar el interés de las personas por aprender la lengua inglesa a través de la gran cantidad de cursos, clases y módulos que se ofrecen sobre la enseñanza de esta lengua. En la Carrera de Lenguas Extranjeras en la Universidad Nacional Autónoma de Honduras ha aumentado la matricula considerablemente en los últimos años lo cual es una evidencia de lo importante que es para las personas el aprendizaje del inglés como lengua extranjera.

El porcentaje más alto de egresados de la Carrera de Lenguas Extranjeras en la UNAH laboran como docentes en las instituciones bilingües de Tegucigalpa y esta es la razón por la cual es importante conocer cuál es el uso que se le da a las Tecnologías de Información y Comunicación en estas instituciones educativas bilingües. Los beneficiarios principales de los resultados de este estudio son los docentes y estudiantes de la Carrera de Lenguas Extranjeras así como toda la población en general interesada en el proceso de enseñanza aprendizaje de esta lengua.

Para la realización de este estudio se realizó una revisión bibliográfica de las últimas investigaciones que se han realizado sobre la misma temática, así como las teorías de diferentes autores en relación al tema de estudio. La información fue recolectada de los centros de educación bilingüe de Tegucigalpa la cual fue la etapa más desafiante de todo el proceso debido a la gran cantidad de tiempo que tomó recopilar la información. El propósito principal de este artículo es mostrar los resultados de este estudio con el fin de que las personas involucradas en el proceso de enseñanza aprendizaje del inglés conozcan las demandas de las instituciones bilingües en lo que al uso de las TIC se refiere para así ofrecer un mejor desempeño laboral y un mejor proceso educativo.

\section{METODOLOGÍA}

a. Diseño: Este estudio se basa en un diseño no experimental, en el que se desarrolla la investigación sin manipular los datos y resultados del mismo. Debido a que no se realiza un experimento, esta investigación es de tipo descriptiva en la que se inten- 
ta describir como las tecnologías de información y comunicación son utilizadas en el proceso de enseñanza del inglés en las instituciones privadas bilingües de Tegucigalpa. Por esta misma razón, no se plantean hipótesis ya que por medio del tipo de investigación descriptivo se intenta obtener una descripción de las variables (Sampieri, 2006).

b. Población: La población de este estudio son todos los estudiantes que pertenecen a estos centros de enseñanza-aprendizaje y los docentes que laboran en estas instituciones. Se solicitó a 30 centros de educación bilingüe de Tegucigalpa para la recolección de la información, sin embargo, la solicitud fue respondida positivamente solo por parte de 10 institutos educativos los cuales son nuestra muestra.

Metodológicamente el tipo de muestreo para este proyecto se denomina no probabilístico ya que la recolección de la información tuvo que someterse a la autorización de las autoridades de las instituciones involucradas en el estudio. A causa del poco acceso a las instituciones bilingües privadas y al poco tiempo brindado para aplicar los cuestionarios, no se pudo realizar un tipo de muestro aleatorio.

La muestra fue un total de 200 participantes todos pertenecientes a diez instituciones bilingües de Tegucigalpa. Se aplicó cuestionarios a 70 docentes; 7 en cada institución, 6 docentes de educación primaria y el docente encargado del aula de informática. Además, se aplicó cuestionarios a una sección de sexto grado en cada institución; la cual comprende un promedio de 13 estudiantes lo que da como resultado 130 estudiantes en los diez centros.

c. Entorno: La información que sirvió de base para responder a los objetivos de este estudio fue recolectada en las siguientes instituciones: International School, La Estancia School, Aldebarán, Gran Comisión, Nashville School, Valencia School, Abundant Life School (Vida Abundante), Arcoíris School, Fuente Divina y CADMUS Academy.

d. Intervenciones: Para la recolección de datos se hizo uso de un cuestionario el cual contiene preguntas abiertas y cerradas. Estas preguntas fueron dirigidas específicamente a los estudiantes y maestros quienes fueron nuestras fuentes de información. Las preguntas en el cuestionario se basaron en nuestras variables de la problemática planteada que son los recursos tecnológicos y el proceso de aprendizaje activo de los estudiantes.

e. Análisis del estudio: Después de recolectar la información, se procedió con la 
elaboración de la base de datos y plantilla en el programa estadístico SPSS para poder interpretar y analizar la información de una manera más agilizada. También se utilizó la herramienta estadística Excel la que permitió la elaboración de tablas y gráficas para que el lector tenga una mejor comprensión de los resultados.

Luego de que los datos fueron tabulados, con las tablas y gráficos, se inició con la redacción del análisis. Para la descripción de los resultados se utilizó frecuencias y porcentajes para un mejor entendimiento de los resultados analizados y de la muestra utilizada.

\section{RESULTADOS}

Gráfico 1. Conocimiento de los docentes sobre el uso de las TIC.

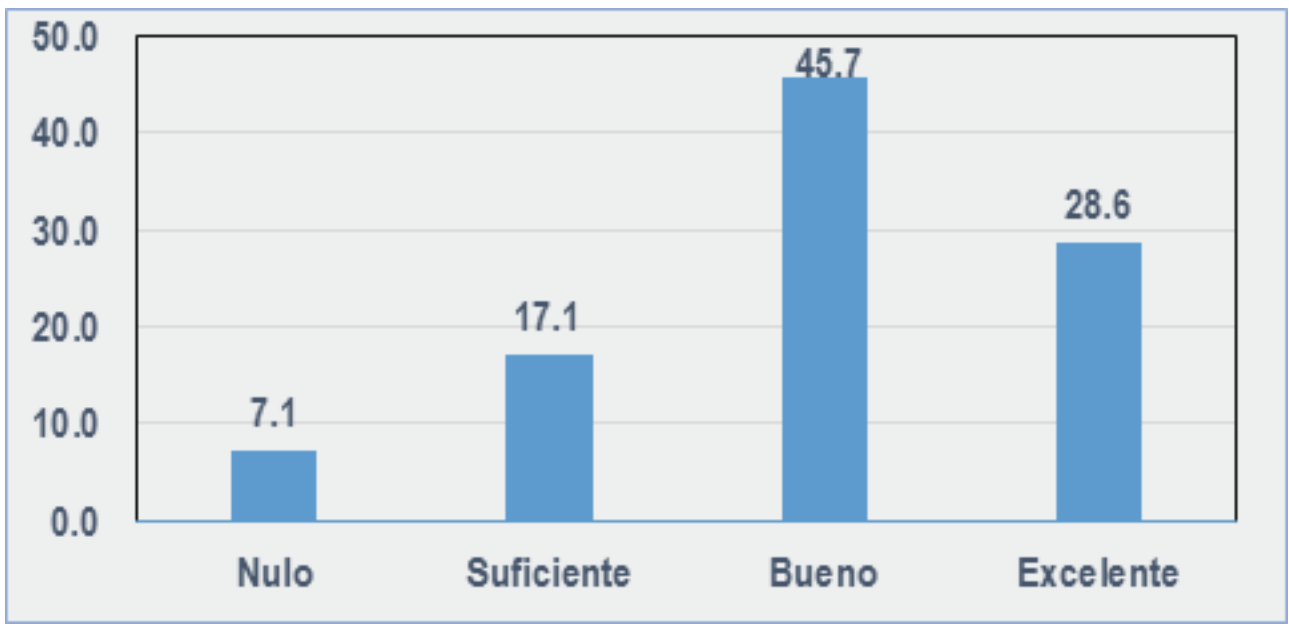

Fuente: Datos obtenidos a través de cuestionarios aplicados a docentes y estudiantes de 10 institutos educativos bilingües de Tegucigalpa.

Los docentes que laboran en las instituciones de educación bilingüe de Tegucigalpa tienen competencias tecnológicas desarrolladas. En el gráfico 1 se puede observar que el $74.3 \%$ de los docentes tienen un nivel aceptable, mientras que el $25.7 \%$ de los maestros necesitan mejorar su nivel de dominio de herramientas y dispositivos tecnológicos. 


\section{Gráfico 2. Capacitación docente en las TIC.}

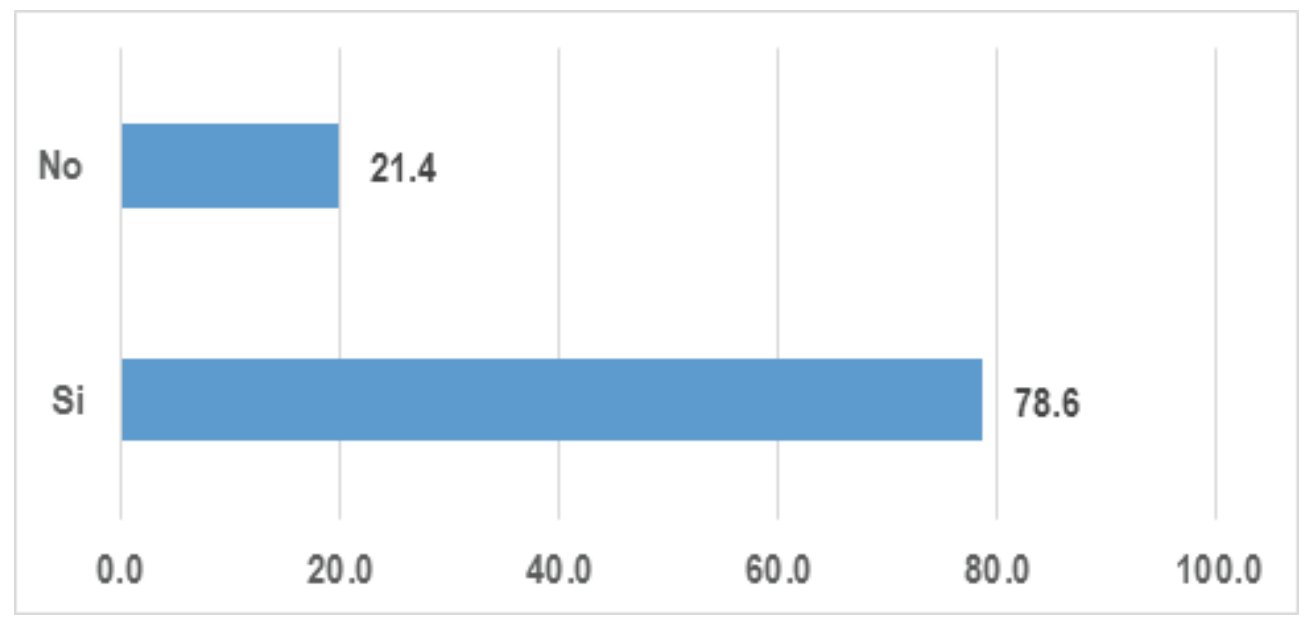

Fuente: Datos obtenidos a través de cuestionarios aplicados a docentes y estudiantes de 10 institutos educativos bilingües de Tegucigalpa.

El buen desempeño laboral docente en los centros educativos bilingües en Tegucigalpa se debe a que los docentes se capacitan regularmente en el uso de las Tecnologías de Información y Comunicación. Sin embargo, el gráfico anterior refleja que el $78.6 \%$ se capacitan en el uso de herramientas tecnológicas, sin embargo, el $21.4 \%$, aproximadamente un quinto (14/70) de los docentes, no se capacitan en el manejo de las TIC para el desarrollo de sus clases.

Gráfico 3. Porcentaje de tiempo que docentes utilizan TIC en sus clases.

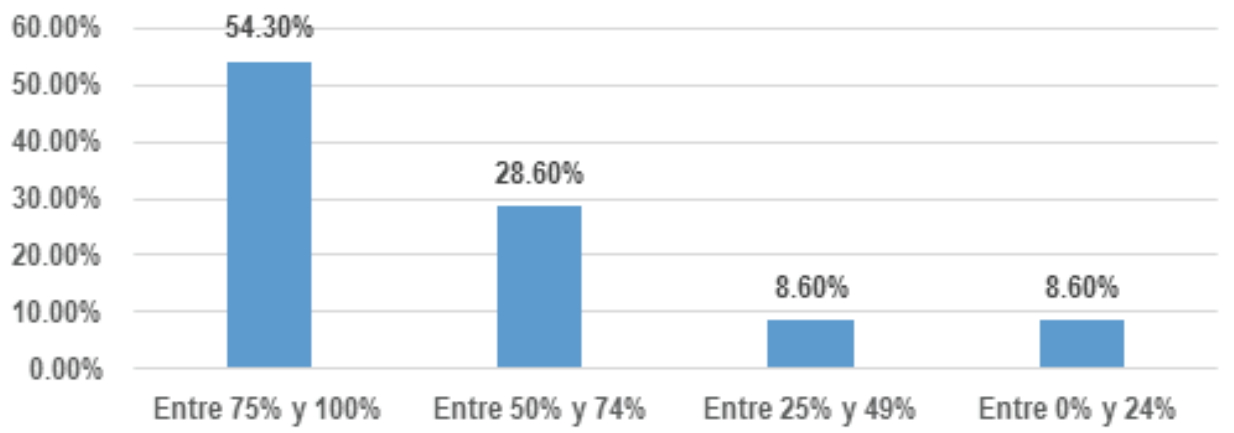

Fuente: Datos obtenidos a través de cuestionarios aplicados a docentes y estudiantes de 10 institutos educativos bilingües de Tegucigalpa. 
En las instituciones de educación bilingüe de Tegucigalpa, el uso de dispositivos tecnológicos es muy regular debido a la facilidad con la que se cuenta en estos centros. El $54.30 \%$ de los encuestados respondieron que estas herramientas tecnológicas son utilizadas entre un 100 y $75 \%$ del tiempo en las clases, mientras que el $28.60 \%$ respondió que las TIC se utilizan entre un 74 y $50 \%$ del tiempo del proceso educativo. Luego se presentan porcentajes más bajos que reflejan menos uso de estas tecnologías.

\section{Gráfico 4. Instituciones que cuentan con dispositivos tecnológicos.}

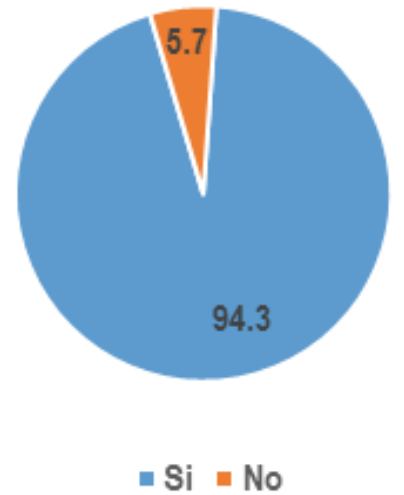

Fuente: Datos obtenidos a través de cuestionarios aplicados a docentes y estudiantes de 10 institutos educativos bilingües de Tegucigalpa.

Las escuelas y colegios bilingües de Tegucigalpa cuentan con dispositivos tecnológicos para brindar una enseñanza de inglés de calidad. El $94.3 \%$ de institutos bilingües involucrados en la investigación cuentan con el equipo tecnológico necesario y solo el $5.7 \%$ no cuentan con estas capacidades tecnológicas. Los encuestados mencionaron que hay necesidad de mayor inversión para la adquisición de más equipo tecnológico y construcción de ambientes virtuales que permitan el buen desempeño de aprendizaje de los estudiantes.

\section{DISCUSIÓN}

Se han realizado una serie de estudios sobre el uso de las tecnologías de información y comunicación en relación al proceso de enseñanza aprendizaje del inglés y se han obtenido diferentes resultados los cuales sirvieron de fundamento teórico para este proyecto. 
La aplicación de las Tecnologías de la Información y Comunicación (TIC) en la enseñanza del inglés como lengua extranjera es una investigación elaborada por Marta Palacios, en el año 2014. Como resultado se obtuvo que el uso de las TIC y en especial de la Tablet PC puede ser muy útil para la enseñanza de una lengua extranjera como el inglés ya que los recursos y aplicaciones para trabajar con este dispositivo en el aula son múltiples y variados (Palacios M.A, 2014).

En 2012 se llevó a cabo un trabajo de investigación sobre la estrategia de enseñanza/aprendizaje en ambientes virtuales en los sistemas de educación a distancia y presencial. Dicha investigación fue dirigida por Gustavo Rodríguez. Como resultado de esta investigación se obtuvo que el $77 \%$ de los encuestados prefieren hoy entornos virtuales para aprender una lengua extranjera (Rodríguez, 2012).

Aparte de estas investigaciones, se han realizado otras que tienen mucha relación con la realización de este estudio como es el caso de Hernández (2007) un proyecto de investigación sobre el CADI, un ambiente virtual para el aprendizaje del idioma inglés en el centro de idiomas de la universidad veracruzana, Lavado Perez, M. (2011) Recursos TIC para ELAO, filologia y traduccion y Morais, P. S. (2012) Estrategia de ensenanza aprendizaje en ambientes virtuales.

En cuanto a los resultados de este estudio, el conocimiento de los docentes sobre el manejo de las TIC es aceptable ya que la mayor parte de los maestros saben que son las TIC y las utilizan en un $75 \%$ del tiempo en sus clases. Además de que un gran porcentaje de docentes han sido capacitados y poseen un nivel bueno de dominio sobre las tecnologías mencionadas. Otra de las razones para decir que los docentes tiene un buen conocimiento de las TIC es que desarrollan actividades con ayuda de la tecnología en sus clases aparte de que han elaborado material didáctico digital. Cabe mencionar que un porcentaje muy mínimo de docentes ha tenido problemas al momento de utilizar estas tecnologías en sus clases y la razón de esto ha sido por causas de falta de equipo tecnológico. Mencionados estos datos, se estima que los docentes de estas instituciones bilingües poseen un buen conocimiento y dominio de las Tecnologías de Información y Comunicación.

Las instituciones de educación bilingüe cuentan con los recursos tecnológicos que permiten el buen aprendizaje de los estudiantes y el dinamismo del proceso educativo. En los gráficos anteriores se puede observar que los docentes tienen acceso a computadoras, proyectores, tabletas, pantallas y otros dispositivos tecnológicos. Así mismo los estudiantes utilizan estos mismos dispositivos aparte de los teléfonos inteligentes y otras herramientas que sirven de apoyo para la realización de las tareas 
académicas. A pesar de la diversidad de dispositivos con que cuentan estas instituciones, los docentes y estudiantes sugieren que se haga una mayor inversión para la adquisición de más equipo tecnológico.

Sobre el impacto de las TIC en el proceso de aprendizaje del inglés, la percepción de los estudiantes se basa en que uno de los beneficios de la integración de la tecnología en el proceso de enseñanza del inglés es el hecho de que la tecnología permite el acceso a todo tipo de información, lo que mejora el aprendizaje de los estudiantes. Otro de los beneficios de las TIC mencionados por los estudiantes, es el dinamismo y alternancia. El uso de estas herramientas permite que los docentes desarrollen diferentes actividades con variedad de estrategias que ayuda a que los estudiantes aprendan de una manera más fácil y divertida por la cual se sienten motivados e interesados. El uso de actividades lúdicas como juegos interactivos, canciones, rompecabezas, crucigramas, sopa de letras, entre otros, es una de las cosas que más llama la atención de los estudiantes.

En el caso de los docentes, se les planteó la misma interrogante y las respuestas más repetitivas se basaron en la actualización del conocimiento que ofrece el uso de la tecnología. Otro de los beneficios mencionados por la mayoría de los docentes es la facilidad de aprendizaje y el desarrollo de habilidades que permiten estas herramientas tecnológicas. De esta manera, las TIC son de beneficio tanto para docentes como para estudiantes ya que mantienen interesados a los estudiantes y permiten que los docentes realicen su trabajo más eficaz y eficientemente y que se alcancen mejores resultados en el proceso de enseñanza del inglés.

De manera general, el impacto de las TIC en el proceso de enseñanza del inglés es muy positivo. La perspectiva de docentes y estudiantes verifica que el uso de estas tecnologías permite que el proceso educativo sea más productivo, así como que el estudiante desarrolle diferentes competencias y habilidades que lo convierten en alguien más preparado capaz de realizar diferentes actividades.

\section{CONCLUSIONES}

1. El uso de las Tecnologías de Información y Comunicación como herramienta para la enseñanza del inglés en los centros de educación bilingüe de Tegucigalpa es un elemento que afecta de manera positiva el aprendizaje de una segunda lengua. De igual manera, el apoyo de estas herramientas tecnológicas permite dinamizar y 
agilizar este proceso como también permite captar la atención e interés de los estudiantes, sin embargo, se debe fomentar más su uso ya que existe un cierto porcentaje de docentes en estas instituciones que no han logrado adaptarse a la utilización de estas herramientas.

2. La mayoría de los docentes encuestados de los centros de educación bilingüe de Tegucigalpa presentan un buen nivel de conocimiento sobre las TIC. Esto se debe a que los docentes han sido capacitados en el uso de estas herramientas lo que permite que los maestros las utilicen en la mayor parte del tiempo en la impartición de las clases y desarrollen actividades pedagógicas que hacen el proceso educativo más provechoso. El buen conocimiento sobre las TIC permite que los docentes puedan elaborar material didáctico digital que puede ser utilizado para diferentes actividades escolares desde pasar la lista de asistencia hasta aplicar un examen.

3. El nivel de acceso a dispositivos tecnológicos por parte de docentes y estudiantes en las instituciones de educación bilingüe es muy satisfactorio. Los docentes y estudiantes de estas instituciones casi en su totalidad disponen de diferentes dispositivos como computadoras, tabletas, proyectores, pantallas inteligentes, celulares inteligentes, entre otros.

4. El impacto de las Tecnologías de Información y Comunicación en el aprendizaje de los estudiantes juega un papel importante debido a la gran diversidad de ventajas que estas ofrecen. Dentro de estas ventajas se encuentra la motivación e interés de los estudiantes de querer aprender a través de estas tecnologías, el desarrollo de actividades lúdicas y el dinamismo y agilización del proceso de aprendizaje del inglés. Por otro lado, una población más pequeña de estas instituciones no está familiarizada con el uso de las Tics y observan que estas herramientas comprenden ciertas desventajas como distracciones en los estudiantes y la falta de conocimiento de estas por parte de los padres de familia para apoyar a sus hijos.

5. Los lineamientos para el diseño de una propuesta pedagógica sobre el uso de las TICs en la enseñanza del inglés en instituciones bilingües son necesarios e importantes para la toma de decisiones con el fin de fortalecer las competencias tecnológicas de los estudiantes de la carrera de lenguas extranjeras. 


\section{RECOMENDACIONES}

1. Capacitar en forma regular a los docentes de las instituciones bilingües de Tegucigalpa con el fin de que se brinde una educación de calidad actualizada tecnológica y pedagógicamente.

2. Promover la utilización de las Tecnologías de Información y Comunicación en el proceso de enseñanza Aprendizaje del inglés así como la motivación e interés de docentes y estudiantes.

3. Fomentar el proceso de enseñanza-aprendizaje del inglés por medio del uso de las TIC en los estudiantes de la Carrera de Lenguas Extranjeras de la UNAH para que estos lo apliquen en las instituciones donde laboran.

4. Desarrollar capacitaciones y talleres sobre el uso de las TIC en la Carrera de Lenguas Extranjeras de la UNAH, tanto para estudiantes como para docentes. Asimismo, se recomienda la integración de estas tecnologías en las clases de la carrera, el uso de materiales digitales y la creación de un portafolio docente digital para los estudiantes próximos a graduarse. Estas recomendaciones se plantean debido a las debilidades identificadas de los docentes en el uso de las herramientas tecnológicas en los centros de educación bilingües de Tegucigalpa.

\section{BIBLIOGRAFÍA}

Hernandez, B. G. (2007). trabajo de grado para optar al titulo de especialista en diseños de ambientes de aprendizaje.Recuperadodehttp://repository.uniminuto.edu:8080/jspui/bitstream/10656/194/1/TEDAA_GarciaHernandezBenjamin

Perez, M. (2011). Mi pagina de recursos TIC para ELAO, filologia y traduccion. Recuperado de $h$ ttp://www.actiweb.es/olgalavado/.

Morais, P. S. (2012). Estrategia de enseñanza-aprendizaje en ambientes virtuales. Artigo 1, volumen (1), 45-54.

Palacios, M. A. (2014). trabajo fin de grado. Obtenido de http://biblioteca.unirioja.es/tfe_e/TFE000715.pdf.

Rodriguez, D. P. (2008). B-Learning como estrategia metológica para mejorar el proceso de enseñanza-aprendizaje de los estudiantes de inglés de la modalidad semipresencia. Tesis doctoral, Universidad Compluetense, Facultad de Edyaca- 
ción. Madrid. Tesis en opcion al titulo de maestro en formacion y capcitacion de recursos humanos. Recuperado de:///D:/Users/Ana\%20Ruth\%20Torres/Downloads/17869.pdf.

Sampieri, R. H. (2006). Metodología de la investigación .México; D.F: MacGraw-HiII/Interamericana. 